\title{
The Radial Dependence of Proton-scale Magnetic Spectral Break in Slow Solar Wind during PSP Encounter 2
}

\author{
Die Duan ${ }^{1,2}$, Trevor A. Bowen ${ }^{2}$ (i), Christopher H. K. Chen ${ }^{3}$ (10), Alfred Mallet ${ }^{2}$, Jiansen He ${ }^{1}$ (D), Stuart D. Bale ${ }^{2,3,4,5}$ (D), \\ Daniel Vech $^{6,7}$ (1) J. C. Kasper ${ }^{6,8}$ (1) Marc Pulupa $^{2}$ (i), John W. Bonnell ${ }^{2}$, Anthony W. Case ${ }^{8}$ (D) Thierry Dudok de Wit ${ }^{9}$, \\ Keith Goetz ${ }^{10}$, Peter R. Harvey ${ }^{2}$, Kelly E. Korreck ${ }^{8}$ (D), Davin Larson ${ }^{2}$, Roberto Livi ${ }^{2}$, Robert J. MacDowall ${ }^{11}$ (D), \\ David M. Malaspina ${ }^{7}$ (D), Michael Stevens ${ }^{8}$, and Phyllis Whittlesey ${ }^{2}$ (D) \\ ${ }^{1}$ School of Earth and Space Sciences, Peking University, Beijing, 100871, People’s Republic of China; dduan@ @ku.edu.cn, jshept@pku.edu.cn \\ ${ }^{2}$ Space Sciences Laboratory, University of California, Berkeley, CA 94720-7450, USA; tbowen@ berkeley.edu \\ ${ }_{3}^{3}$ School of Physics and Astronomy, Queen Mary University of London, London E1 4NS, UK \\ ${ }^{4}$ Physics Department, University of California, Berkeley, CA 94720-7300, USA \\ ${ }^{5}$ The Blackett Laboratory, Imperial College London, London, SW7 2AZ, UK \\ ${ }_{7}^{6}$ Climate and Space Sciences and Engineering, University of Michigan, Ann Arbor, MI 48109, USA \\ ${ }^{7}$ Laboratory for Atmospheric and Space Physics, University of Colorado, Boulder, CO 80303, USA \\ ${ }^{8}$ Smithsonian Astrophysical Observatory, Cambridge, MA 02138, USA \\ ${ }^{9}$ LPC2E, CNRS and University of Orléans, Orléans, France \\ ${ }^{10}$ School of Physics and Astronomy, University of Minnesota, Minneapolis, MN 55455, USA \\ ${ }^{11}$ Solar System Exploration Division, NASA/Goddard Space Flight Center, Greenbelt, MD 20771, USA \\ Received 2019 September 19; revised 2019 December 5; accepted 2019 December 14; published 2020 February 3
}

\begin{abstract}
Magnetic field fluctuations in the solar wind are commonly observed to follow a power-law spectrum. Near protonkinetic scales, a spectral break occurs that is commonly interpreted as a transition to kinetic turbulence. However, this transition is not yet entirely understood. By studying the scaling of the break with various plasma properties, it may be possible to constrain the processes leading to the onset of kinetic turbulence. Using data from the Parker Solar Probe, we measure the proton-scale break over a range of heliocentric distances, enabling a measurement of the transition from inertial to kinetic-scale turbulence under various plasma conditions. We find that the break frequency $f_{b}$ increases as the heliocentric distance $r$ decreases in the slow solar wind following a power law of $f_{b} \sim r^{-1.11}$. We also compare this to the characteristic plasma ion scales to relate the break to the possible physical mechanisms occurring at this scale. The ratio $f_{b} / f_{c}\left(f_{c}\right.$ for Doppler-shifted ion cyclotron resonance scale) is close to unity and almost independent of plasma $\beta_{p}$. While $f_{b} / f_{\rho}$ ( $f_{\rho}$ for Doppler-shifted proton thermal gyroradius) increases with $\beta_{p}$ approaching to unity at larger $\beta_{p}, f_{b} / f_{d}\left(f_{d}\right.$ for Doppler-shifted proton inertial length) decreases with $\beta_{p}$ from unity at small $\beta_{p}$. Due to the large comparable Alfvén and solar wind speeds, we analyze these results using both the standard and modified Taylor hypotheses, demonstrating the robust statistical results.
\end{abstract}

Unified Astronomy Thesaurus concepts: Solar wind (1534); Interplanetary turbulence (830); Space plasmas (1544); Alfven waves (23)

\section{Introduction}

Understanding processes of kinetic dissipation in magnetized plasma is essential for explaining the physical origin and evolution of the solar wind. Observationally, the power spectral density (PSD) of the magnetic field fluctuations is commonly divided into two regimes separated by a spectral break. The lower frequencies, corresponding to larger physical scales, correspond to magnetohydrodynamic (MHD) fluctuations, with an inertial range of turbulence similar to the Kolmogorov $f^{-5 / 3}$ power-law spectrum. In the high-frequency range, the power spectra is observed to steepen with a spectral index of between -2 to -4 (Bruno \& Carbone 2013; Kiyani et al. 2015; Chen 2016). These scales are thought to correspond to scales in which the MHD approximation is no longer valid, and the kinetic effects of the protons should be considered (Alexandrova et al. 2009). However, the specific processes occurring in the kinetic range have not been determined, with significant debate regarding the nature of the fluctuations and the relevant nonlinear processes (Howes 2017).

The steepening of the spectral index possibly implies that cascaded energy at the end of MHD scale may be gradually dissipated or develop into a dispersive kinetic turbulence.
Observationally, the solar wind expands non-adiabatically, indicating that in situ heating must occur. Dissipation of the inertial-range turbulence is one source of energy capable of proton heating, though there are multiple mechanisms that may lead to dissipation (Marsch 2006). Kinetic Alfvén waves (KAW) could start to dissipate via Landau damping since the scale of the proton gyroradius is $\rho_{p}=v_{\text {th, } p} / \Omega_{p}$, where $v_{\text {th }, p}$ is the thermal velocity of the proton, $\Omega_{p}=e B / m_{p}$ is the proton gyrofrequency, $e$ is the elementary charge, $B$ is the mean magnetic field, and $m_{p}$ is the mass of the proton (Leamon et al. 1999; Schekochihin et al. 2009). Stochastic proton heating is also a possible dissipation mechanism at scales near $\rho_{p}$. The ions could be heated perpendicularly when the amplitude of the gyroscale fluctuations is large (Chandran et al. 2010; Bourouaine \& Chandran 2013; Vech et al. 2017; Martinović et al. 2019). The proton inertial length of $d_{p}=v_{A} / \Omega_{p}$ is another important scale, where $v_{A}=B / \sqrt{\mu_{0} n_{p} m_{p}}$ is the Alfvén speed, with $\mu_{0}$ being the vacuum magnetic permeability and $n_{p}$ being the proton density. The inertial length corresponds to the scale at which electrons can decouple from protons and it may limit the size of small-scale current sheets formed through nonlinear turbulent processes, which in turn may dissipate energy 
through magnetic reconnection (Leamon et al. 2000; Dmitruk et al. 2004; Vasquez et al. 2007).

Alfvén waves with quasi-parallel propagation at a relatively higher frequency may dissipate through cyclotron resonance damping. For parallel propagating Alfvén waves, the damping will occur at the (parallel) wavenumber corresponding to the cyclotron resonance, $k_{c}=\Omega_{p} /\left(v_{A}+v_{\text {th }, p}\right)$ (Leamon et al. 1998). Studies of anisotropy in solar wind turbulence using the method introduced by Cho \& Vishniac (2000) and Horbury et al. (2008) suggest that the inertial range is highly anisotropic near the kinetic break with $k_{\perp} \gg k_{\|}$, such that most of the energy is contained in perpendicular fluctuations that do not have parallel wavenumbers resonant with parallel cyclotron waves (Chen et al. 2010). The 2D PSD distribution $\left(k_{\|}, k_{\perp}\right)$, as reconstructed with the tomography method based on Fourier projection-slice theorem, reveals the dominance of oblique propagation of Alfvénic fluctuations extending its power ridge to higher $k_{\perp}$ and also higher $k_{\|}$, which indicates the existence of oblique Alfvén-cyclotron waves (He et al. 2013; Yan et al. 2016).

Alternatively, the change of the spectral slope may indicate a transition from a cascade of non-dispersive Alfvén waves to a cascade of dispersive KAW around the scale of $k_{\perp} \rho_{p} \sim 1$ (Bale et al. 2005; Howes et al. 2008; Schekochihin et al. 2009). It has been additionally suggested that a cascade of whistler modes or magnetosonic waves may develop at kinetic scales (Stawicki et al. 2001; Gary \& Smith 2009). Furthermore, the inclusion of the Hall term in the MHD approximation has been proposed as the source of the break at scales of $d_{p} k_{\perp} \sim 1$ (Galtier 2006). Mallet et al. (2017) and Loureiro \& Boldyrev (2017) suggest that the inertial-range turbulence could generate sheet-like turbulent structures, which could be disrupted by reconnection below a disruption scale intermediate to $d_{p}$ and $\rho_{p}$.

Given the number of potential mechanisms that generate a spectral break, and the relatively narrow range in the physical scales predicted, distinguishing between these various mechanisms using empirical measurements has proven to be a difficult task (Markovskii et al. 2008). Furthermore, these different physical processes may occur simultaneously in the solar wind, complicating efforts to quantify their relative contributions (Verscharen et al. 2019).

Many previous studies have explored the transition from inertial to kinetic-scale physical processes through both observations and simulations, although no consensus has been reached. Observationally, the mechanisms that lead to spectral steepening may be constrained by investigating the dependence of the spectral break frequency on various plasma parameters. For example, the $\beta_{p}$ dependence of the break scale has been studied at 1 au using Wind data, where $\beta_{p}=\rho_{p}^{2} / d_{p}^{2}$ is the ratio of proton thermal pressure to magnetic pressure. For example, Chen et al. (2014) found the break frequency $\left(f_{b}\right)$ close to $f_{d}$ at $\beta_{p} \ll 1$ and close to $f_{\rho}$ at $\beta_{p} \gg 1$, where $f_{d}=v_{\mathrm{sw}} /\left(2 \pi d_{p}\right)$ and $f_{\rho}=v_{\mathrm{sw}} /\left(2 \pi \rho_{p}\right)$ are the frequencies corresponding to the spatial scales $d_{p}$ and $\rho_{p}$ in the spacecraft frame under the Taylor hypothesis, which approximates the observed time evolution of fluctuations in the spacecraft frame as spatial structures advected at the solar wind speed, $v_{\mathrm{sw}}$. Numerical 2D-hybrid simulations found similar $\beta_{p}$ dependence (Franci et al. 2016). Wang et al. (2018) found $f_{b} / f_{d}$ is statistically independent with $\beta_{p}$ of $0.1<\beta_{p}<1.3$ plasma. Woodham et al. (2018) and Duan et al. (2018) suggest that the spectral break is best associated with the proton cyclotron scale of $f_{c}=v_{\mathrm{sw}} k_{c} /(2 \pi)$. Vech et al. (2018) proposed that the break may be caused by magnetic reconnection at a disruption scale intermediate to $d_{p}$ and $\rho_{p}$ as predicted in Mallet et al. (2017). The spectral break is found to be independent of $\theta_{\mathrm{VB}}$, which is the angle between solar wind velocity and the magnetic field, indicating that the spectral break seems to be isotropic in the wavenumber space (Duan et al. 2018). Duan et al. (2018) further proposed and illustrated that the breakdown of the magnetic frozen-in condition in wavenumber space, as a combination of dissipation and dispersion effects, could be a more isotropic explanation compared to the dissipation or the dispersion alone.

Several studies investigated the break scale at different heliocentric distances and its relation with plasma scales. Perri et al. (2010) suggested the break frequency did not show any remarkable evolution between 0.3 and 4.9 au based on observations from MErcury Surface, Space ENvironment, GEochemistry, and Ranging and Ulysess. Bourouaine et al. (2012) also found the break frequency $f_{b}$ does not change significantly from 0.3 to 0.9 au from Helios 2 , and $f_{b}$ follows $f_{d}$ assuming a 2D turbulence model. Bruno \& Trenchi (2014a) found that the break moves to higher frequencies as the heliocentric distance decreases, finding agreement with the proton cyclotron resonance scale between 0.42 and 5.3 au. While many previous studies have focused on the radial behavior of the spectral break in the fast solar wind, the scaling of the spectral break in the slow wind has not been investigated.

NASA' s Parker Solar Probe (PSP) provides a set of in situ instruments capable of constraining the kinetic processes that contribute to heating and acceleration in the corona and nascent solar wind (Bale et al. 2016; Fox et al. 2016; Kasper et al. 2016; He \& Tian 2019). This paper provides a statistical analysis of the behavior of the proton-scale spectral break observed by the PSP between 0.17 and 0.63 au and its radial dependence in the slow solar wind. By measuring the radial dependence of the break, we are able to compare the location of the spectral break with various physical scales under a range of plasma conditions, enabling an investigation into the mechanisms behind spectral steepening of the kinetic range.

\section{Data and Method}

We analyze 26 days of data from PSP during the cruise phase of the second orbit of PSP from 2019 March 10 to April 5. Data on March 16 were excluded as the time resolution of the magnetic field is not sufficient to resolve the spectral break. During the period, PSP covers the distance between 0.63 au (March 10) and 0.17 au (April 5) from the Sun. Magnetic field measurements on PSP are made by the FIELDS/fluxgate MAGnetometer (MAG; Bale et al. 2016). Measurements of the solar wind speed, thermal speed, and proton density by the Solar Wind Electrons Alphas and Protons Investigator (SWEAP)/Solar Probe Cup (SPC) instrument are used to compute plasma scales (Kasper et al. 2016). Sample rates of FIELDS and SWEAP data vary between the different mission phases and encounters. Between 2019 March 10 and 31, PSP was in the cruise phase with a low-cadence (MAG 9.2 Hz and SPC $0.036 \mathrm{~Hz}$ ) sample rate. From 2019 March 31 to April 4, the mission was in the encounter phase near perihelion, and higher-cadence measurements are obtained (MAG $149 \mathrm{~Hz}$ and SPC $5 \mathrm{~Hz}$ ). Figure 1(a) shows an overview of the trajectory of the PSP in the rotating Carrington heliographic frame. For the majority of the orbit, PSP is in slow solar wind $\left(v_{\mathrm{SW}}<500 \mathrm{~km} \mathrm{~s}^{-1}\right)$. There are no intervals with average $v_{\mathrm{SW}}>500 \mathrm{~km} \mathrm{~s}^{-1}$. Figure 1(b) shows $\beta_{p}$ as a 
(a) PSP in Carrinton Frame

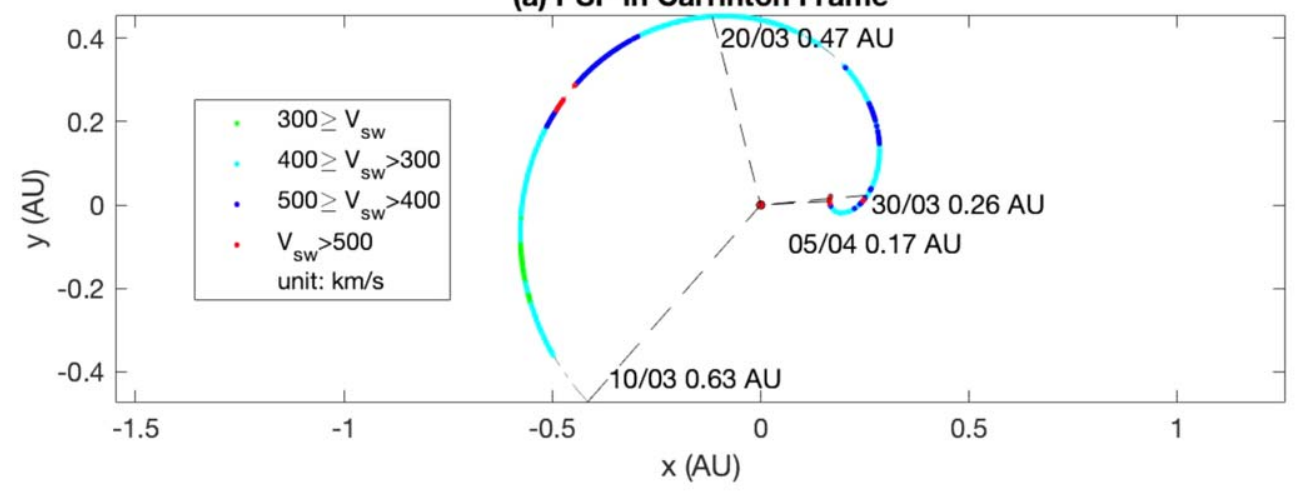

(b) plasma $\beta_{\mathbf{p}}$

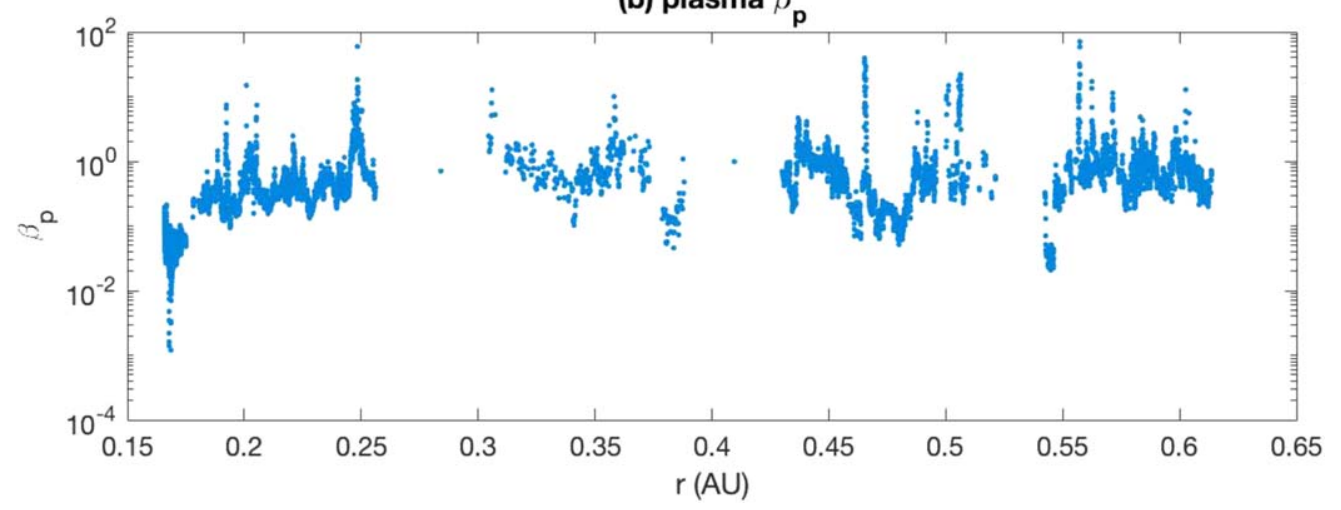

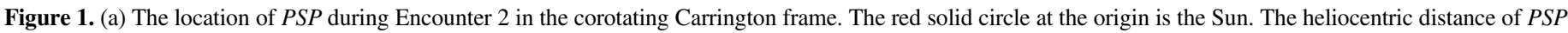

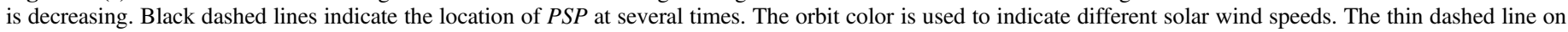
the orbit means the SPC data was unavailable. (b) $\beta_{p}$ at different distances. Blank regions indicate unavailable data.

Table 1

The Selected Fitting Frequency Interval for the Dissipation Range

\begin{tabular}{lcc}
\hline \hline Date & $r(\mathrm{au})$ & Frequency $(\mathrm{Hz})$ \\
\hline Mar 10-11 & $0.60-0.63$ & $0.8-1.4$ \\
Mar 12-15 & $0.54-0.60$ & $0.9-1.4$ \\
Mar 17-19 & $0.47-0.52$ & $0.9-1.5$ \\
Mar 20-24 & $0.37-0.47$ & $1.2-2.2$ \\
Mar 25-28 & $0.28-0.37$ & $1.5-2.5$ \\
Mar 29-30 & $0.23-0.28$ & $1.5-3$ \\
Mar 31-Apr 5 & $0.17-0.23$ & $2-5$ \\
\hline
\end{tabular}

function of the heliocentric distance $r$. As the distance between $P S P$ and the Sun decreases, the proton plasma $\beta$ also decreases due to the increasing strength of the magnetic field: typically, $\beta_{p}<1$.

The trace PSD is estimated by applying a continuous moving window transform on the vector magnetic field. The 26 day interval is divided into partially overlapping 10 minute segments. The beginnings of each adjacent segments are 2.5 minutes apart (overlapping 75\%). A Hanning window is used to reduced spectral leakage in each segment. For each segment, the power spectrum is taken using an ensemble average of five adjacent segments. Each PSD actually correspond to data of 20 minutes.

To locate the proton-scale spectral break, we employed the method of Bruno \& Trenchi (2014a) and Wang et al. (2018).
Two frequency ranges at either end of the spectrum are a priori selected as the inertial (between 0.1 and $0.5 \mathrm{~Hz}$ ) and dissipation ranges. Table 1 highlights the range of frequencies for the dissipation spectra over the orbit. A least-squares linear fit of a power law in logarithmic space is performed on the data over each range. The break frequency $f_{b}$ is defined as the intersection of the two fitting lines. Because the range of spacecraft frequencies that correspond to the dissipation range changes with the heliocentric distance, the range over which the fit is performed is varied throughout the orbit. Additionally, spectral flattening is observed when the amplitude of the turbulent fluctuations reaches the noise level of the MAG $\left(10^{-3} \sim\right.$ $\left.10^{-4} \mathrm{nT}^{2} \mathrm{~Hz}^{-1}\right)$. Because of the decreasing strength of the fluctuations at larger distances, the noise floor is reached at lower frequencies in the cruise data.

Figure 2 shows an example of power density spectra at several distances with measured spectral indices and breaks. At larger distances, the spectral break shifts to a lower spacecraft frame frequency. The top three PSDs show a typical inertialrange slope of $-5 / 3<\alpha_{1}<-3 / 2$ and a dissipation range slope of $\alpha_{2} \approx-4$. The spectra from 0.62 au does not show an obvious break between two power-law spectra. Additionally, the inertial-range spectral index is somewhat steeper than what is typically observed. This shape has been previously reported by Bruno \& Trenchi (2014b) in slow winds. Bowen et al. (2018) demonstrates that the presence of steep magnetic spectra (i.e., $\alpha_{1} \sim-2$ ) likely corresponds to observations of intermittency in the turbulent fluctuations. 


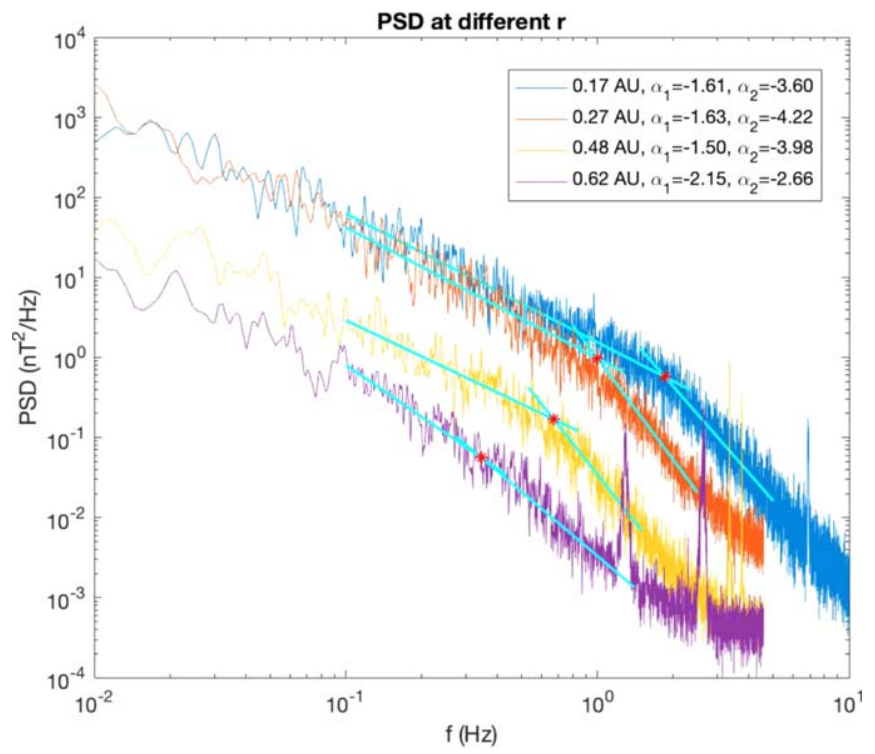

Figure 2. Examples of the PSDs of magnetic field fluctuations at several heliocentric distances. The cyan lines indicate fitted power-law spectra. The red stars are intersections of the fitted lines and are defined as the break frequency $f_{b}$. The fitted inertial-range index $\alpha_{1}$ and fitted dissipation range index $\alpha_{2}$ are shown in the legend.

We removed several intervals with spectral features peaked at ion scales, which results in a deviation from power-law distributions. The presence of these features is likely a secondary population of ion cyclotron waves (Bowen et al. 2020). To systematically control for effects from secondary population of fluctuations, we only accept spectra that fall within a range of spectral indices statistically consistent with known turbulent scalings, $-2.5<\alpha_{1}<-1.2$ and $\alpha_{1}>\alpha_{2}$. In total, 14,820 intervals were obtained with 10,724 of them returning $\alpha_{1}$ and $\alpha_{2}$ within our constrained bounds. Of these intervals, 5194 have corresponding particle data. Mean values of $v_{\mathrm{sw}}, v_{\mathrm{th}, p}$, and $n_{p}$ are averaged over each of intervals. $k_{c}, d_{p}, \rho_{p}$, and $\beta_{p}$ are calculated from the plasma data. We find that $\beta_{p}<1$ in 4479 intervals and $\beta_{p}>1$ in 715 intervals.

Under the Taylor hypothesis, the relation between the wavevector of the fluctuation $\boldsymbol{k}$ and the corresponding frequency $f$ in the spacecraft frame is $2 \pi f=\boldsymbol{k} \cdot \boldsymbol{v}_{\mathrm{sw}}$. Several possible assumptions can possibly made for simplifying the wavevector direction relative to the solar wind flow. If the fluctuations propagate along the solar wind direction, $2 \pi f=k$ $v_{\mathrm{sw}}$. If the fluctuations propagate parallel to the mean magnetic field direction, $2 \pi f=k v_{\mathrm{sw}} \cos \left(\theta_{V B}\right)$. If quasi-2D turbulence with dominant perpendicular fluctuations is assumed, then $\omega=k_{\perp} v_{\mathrm{sw}} \sin \left(\theta_{V B}\right) \cos (\phi)$, where $\phi$ is the angle between the wavevector and the $\left(\boldsymbol{v}_{\mathrm{sw}}, \boldsymbol{B}\right)$ plane (Bourouaine et al. 2012). Duan et al. (2018) found that the spectral break frequency is invariant with the magnetic field's orientation, suggesting that the approximation of $2 \pi f=k v_{\mathrm{sw}}$ is appropriate. The corresponding frequencies for the physical scales are $f_{c}=v_{\mathrm{sw}} k_{c} /(2 \pi)$, $f_{\rho}=v_{S W} /(2 \pi \rho)$, and $f_{d}=v_{\mathrm{sw}} /\left(2 \pi d_{p}\right)$.

Due to the comparable Alfvén and solar wind, and spacecraft speeds, it is unclear whether the Taylor hypothesis is valid for PSP observations during its perihelion (Narita et al. 2013; Bourouaine \& Perez 2018, 2019; Chhiber et al. 2019). Recent

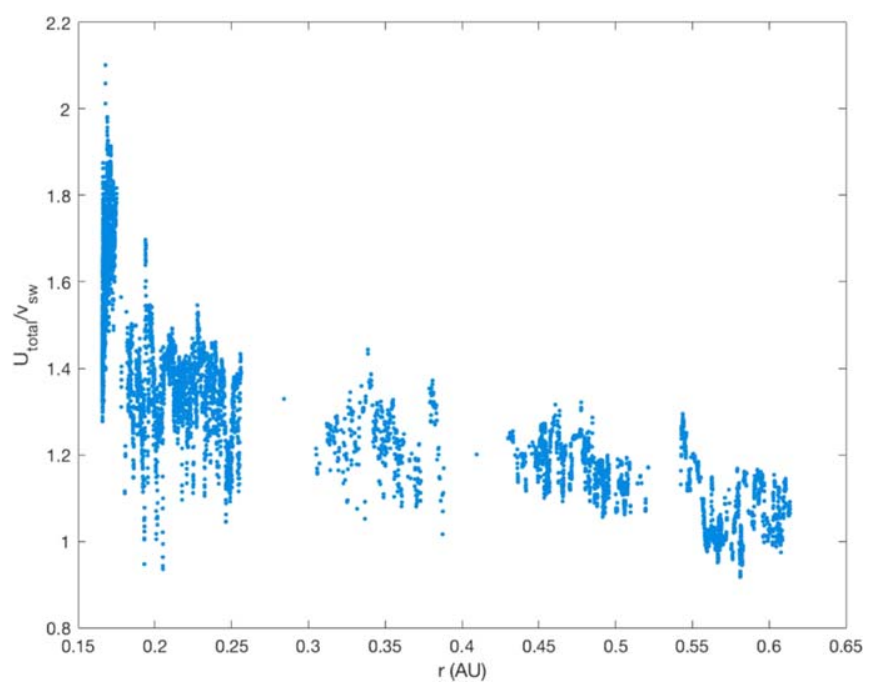

Figure 3. Ratio of $U_{\text {total }}$ to $v_{\mathrm{sc}}$ during the cases.

work from A. Chaspis (2020, in preparation) suggests the Taylor hypothesis may not be applicable when PSP is below 40 solar radii $(0.19 \mathrm{au})$. To verify our results against the assumption of the Taylor hypothesis, we apply an analysis of the proton break scaling to the modified Taylor hypothesis: $2 \pi f^{*}=\boldsymbol{k} \cdot \boldsymbol{U}_{\text {total }}$ (Klein et al. 2015). Here, $U_{\text {total }}=v_{\mathrm{sw}}+\boldsymbol{v}_{A}-\boldsymbol{v}_{\mathrm{sc}}$, where $\boldsymbol{v}_{\mathrm{sc}}$ is the velocity of the PSP. The modified Taylor hypothesis assumes that the anti-sunward propagating fluctuations are approximately frozen into a frame with velocity $\boldsymbol{U}_{\text {total }}$ if the fluctuations do not grow or damp significantly when passing over the spacecraft. The modified corresponding characteristic frequencies are $f_{c}^{*}$ $=U_{\text {total }} k_{c} /(2 \pi), f_{d}^{*}=U_{\text {total }} /\left(2 \pi d_{p}\right)$, and $f_{\rho}^{*}=U_{\text {total }} /\left(2 \pi \rho_{p}\right)$, where $U_{\text {total }}=\left|\boldsymbol{U}_{\text {total }}\right|$. Figure 3 shows $U_{\text {total }} / v_{\text {sw }}$ during our cases. The ratio is almost greater than 1 ( $97 \%$ of cases), making the modified characteristic frequencies smaller, especially below $0.19 \mathrm{au}$. This modified Taylor hypothesis could hold as the outward-propagating fluctuations are dominant near the perihelion (Chen et al. 2020).

\section{Results}

Figure 4(a) shows the distribution of the break frequency $f_{b}$ with the heliocentric distance $r$. Figure 4(b) shows the distribution of $f_{b}$ with $\beta_{p}$. The data are binned in a $20 \times 20$ grid in $\log -\log$ space. There is large variation in $f_{b}$ and a clear radial dependence with a power law of $f_{b} \sim r^{-1.11 \pm 0.01}$. A Pearson correlation coefficient is calculated with PCC $\left(r, f_{b}\right)=-0.81$, and a Spearman correlation coefficient of $\operatorname{SCC}\left(r, f_{b}\right)=-0.84$. This result is similar to the scalings in the fast solar wind suggested by Bruno \& Trenchi (2014a). This radial trend is also consistent with the outer-scale break of the PSD (Chen et al. 2020).

The $f_{b}$ shows a weak dependence with $v_{\mathrm{sw}}$ with $\mathrm{PCC}\left(v_{\mathrm{sw}}, f_{b}\right)=$ 0.14 and $\operatorname{SCC}\left(v_{\mathrm{sw}}, f_{b}\right)=0.10 . f_{b}$ also decreases with $\beta_{p}$; PCC $\left(\beta_{p}, f_{b}\right)=-0.49$ and $\operatorname{SCC}\left(\beta_{p}, f_{b}\right)=-0.51$.

To investigate the correlation between $f_{b}$ and physical plasma scales, we calculated average $f_{\rho}, f_{d}$, and $f_{c}$ for each interval having the measurement of particle data. Table 1 shows that $f_{b}$ is correlated with all of these scales to a similar 
(a)

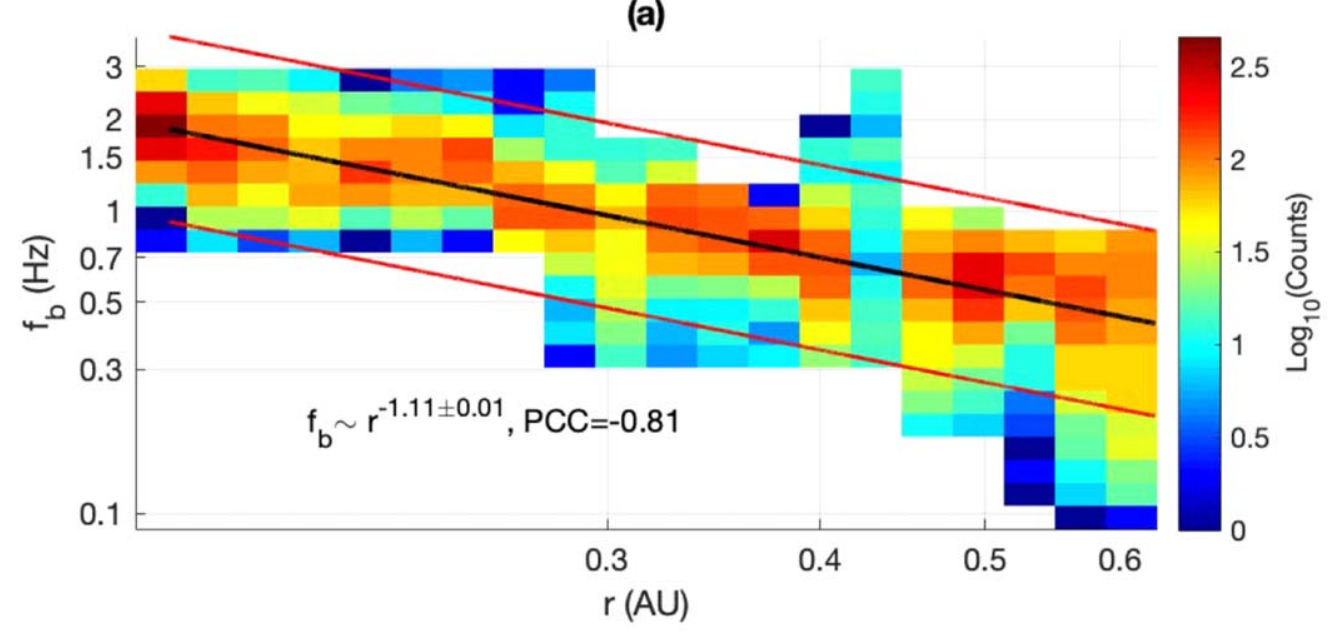

(b)

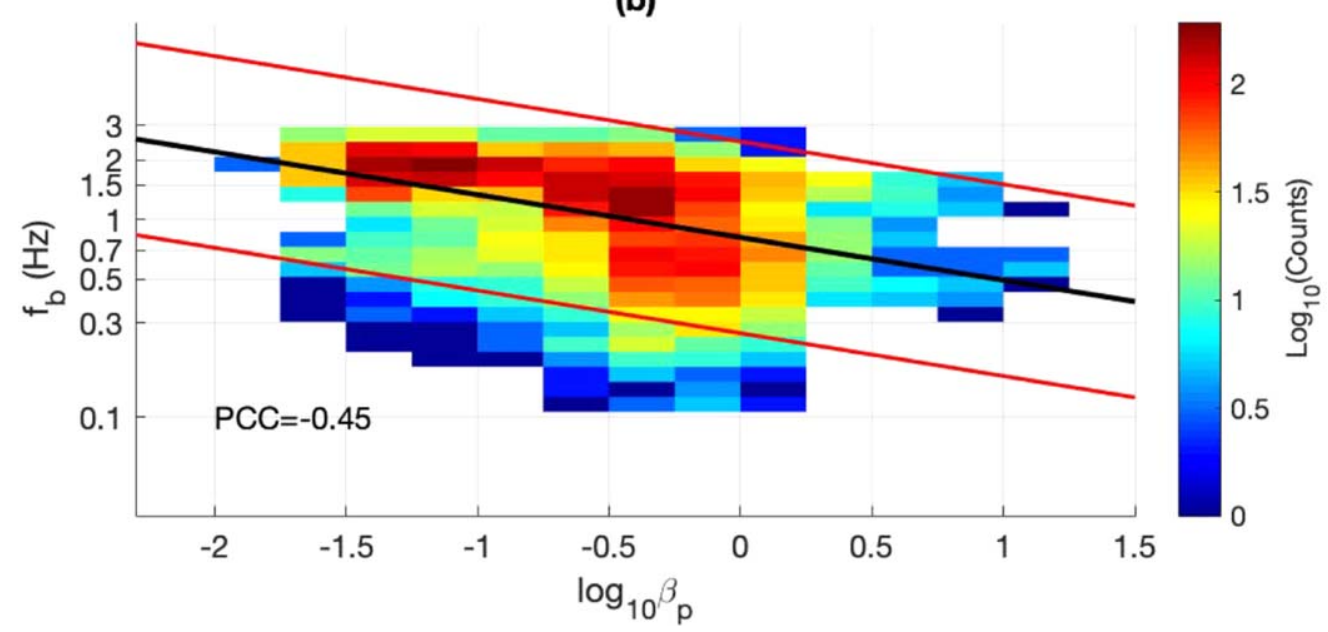

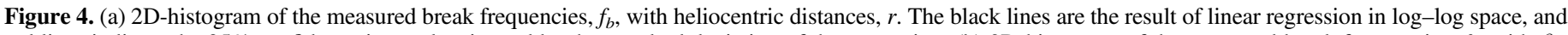

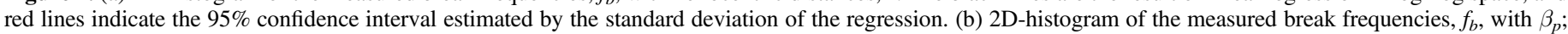

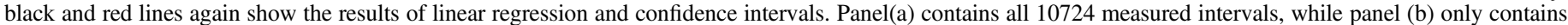
intervals with available SPC data.

degree. It is accordingly difficult to uniquely distinguish the scale that best represents the break frequency.

The ratio of $f_{b}$ to these characteristic frequencies are calculated and illustrated in Figures 5(a)-(c). The data is again binned in a $20 \times 20$ grid in $\log -\log$ space. The average and the stand deviation inside each bin are illustrated with blue lines. The average and the standard deviation of each ratio over all of the data is $0.87 \pm 0.34\left(f_{b} / f_{c}\right), 0.56 \pm 0.24\left(f_{b} / f_{d}\right)$, and $0.32 \pm 0.22\left(f_{b} / f_{\rho}\right)$. The spectral break occurs nearest the cyclotron resonance frequency. The average $f_{b} / f_{c}$ is the largest in each bin. $f_{b} / f_{c}$ and $f_{b} / f_{d}$ decrease as the distance become larger, while $f_{b} / f_{\rho}$ is the opposite. Panels (d)-(f) show the ratio of modified frequencies. We get the same result assuming the modified Taylor hypothesis.

Figure 6 shows the $\beta_{p}$ dependence of the ratios. The result is similar to Chen et al. (2014). $f_{b}$ locates around $f_{d}\left(f_{b} / f_{d} \approx 1\right)$ where $\beta_{p} \ll 1$, while $f_{b}$ locates around $f_{\rho}\left(f_{b} / f_{\rho} \approx 1\right)$ where $\beta_{p} \gg 1$. $f_{b}$ approaches $f_{c}\left(f_{b} / f_{c} \approx 1\right)$ for all $\beta_{p}$. The modified ratios have the similar trends. The correlation coefficients are shown in Table 2. As $f_{c}=\left(1 / f_{d}+1 / f_{\rho}\right)^{-1}$, the $f_{c}$ is close to the smaller of $f_{d}$ and $f_{\rho}$. Our result could not distinguish the behavior of the different possibilities.

\section{Conclusion and Discussion}

We investigated the radial and $\beta_{p}$ dependence of the observed proton-scale magnetic spectral break frequency $f_{b}$ in the slow solar wind from $0.17 \mathrm{au}<r<0.63 \mathrm{au}$. Additionally, we compared the break scale with the spacecraft frequencies corresponding to the cyclotron resonance, $f_{c}$; the proton gyroscale, $f_{\rho}$; and the proton inertial scale, $f_{d}$, over the range of heliocentric distances, $r$. The results show that the break frequency follows a power law of $f_{b} \propto r^{-1.11}$. We find that the break frequency has mild correlation with all of the three plasma characteristic scales. There is no clearly statistic difference between the result from the plain and the modified Taylor hypothesis. However, $f_{b} / f_{c}$ is closest to unity over the full range of distances covered. Nevertheless, since the predicted breaks scales are typically only defined to order unity, it is difficult to distinguish them at the moderate values of $\beta_{p}$ observed by PSP to date.

This work provides the first measurement of the radial scaling of the proton-scale break in the slow solar wind in the inner heliosphere down to $0.17 \mathrm{au}$. The slow solar wind break manifests a radial dependence similar to the fast wind, with the spectral break occurring around the ion cyclotron resonance 

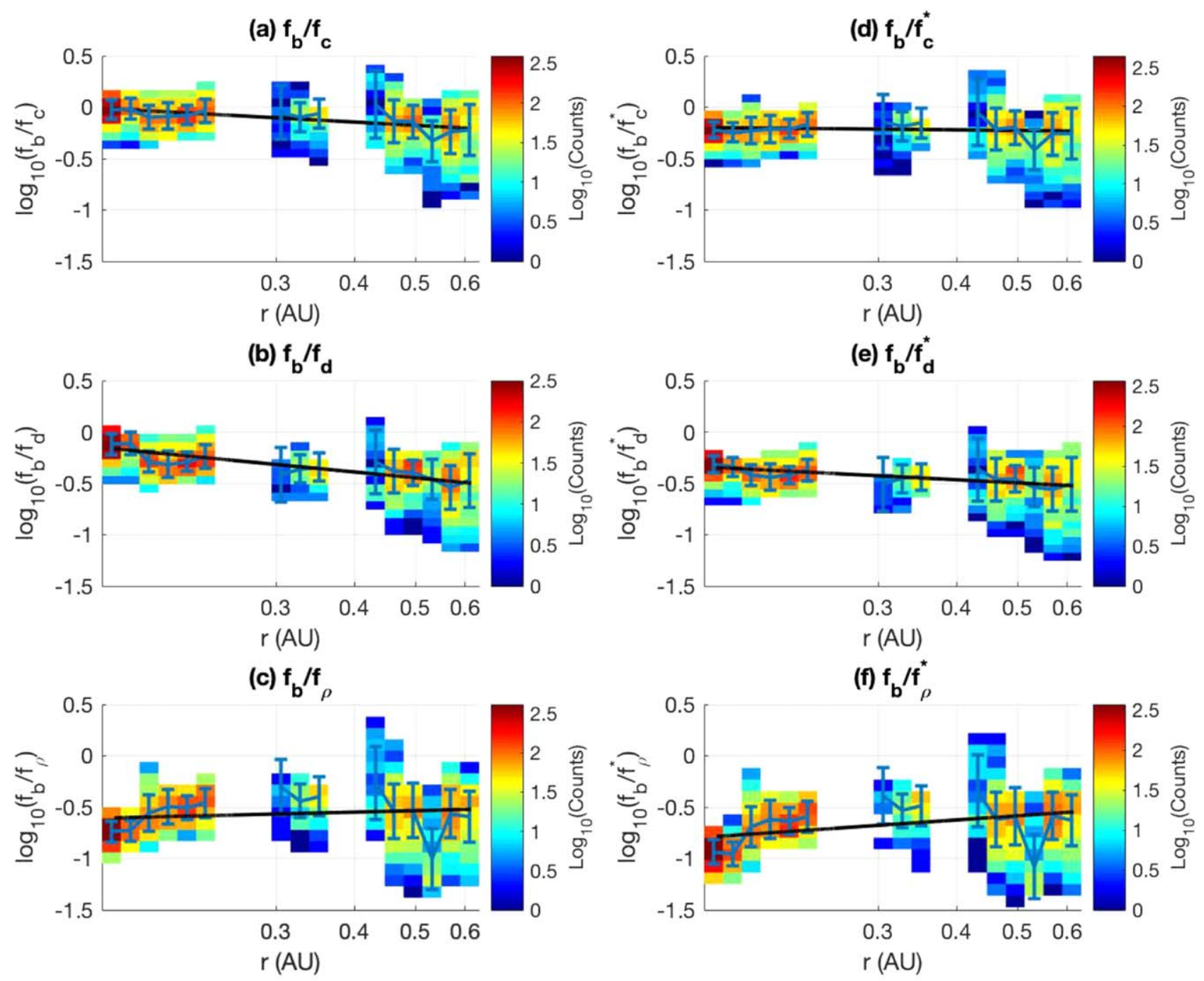

Figure 5. The 2D-histogram of the distribution of the occurrence of (a) $\log _{10}\left(f_{b} / f_{c}\right)$, (b) $\log _{10}\left(f_{b} / f_{d}\right),\left(\right.$ c) $\log _{10}\left(f_{b} / f_{\rho}\right),\left(\right.$ d) $\log _{10}\left(f_{b} / f_{c}^{*}\right),\left(\right.$ e) $\log _{10}\left(f_{b} / f_{d}^{*}\right), \operatorname{and}(\mathrm{f})$ $\log _{10}\left(f_{b} / f_{\rho}^{*}\right)$ over the heliocentric distance $r$, respectively. The starred frequencies are the corresponding frequencies from the modified Taylor hypothesis. The black lines are the linear fitting with least-squares method. The averages and standard deviations of each $r$ bin are plotted as blue lines.

scales (Bruno \& Trenchi 2014a). This suggests that cyclotron resonance may be an important process in the slow solar wind, similar to observations at $1 \mathrm{au}$, although the anisotropy of the turbulence complicates a simple picture of parallel-wavenumber cyclotron damping of Alfvén waves.

The ratio $f_{b} / f_{c}$ approaches unity near the Sun, which may be due to the increased activity of the solar wind plasma close to the Sun generating ion cyclotron waves (Bowen et al. 2020). Because $f_{b} / f_{c}$ deviates slightly from unity (less than unity) in the slow solar wind at 1 au (Woodham et al. 2018) and because $f_{b} / f_{c}$ increases slightly with decreasing heliocentric distances in the slow solar wind, it seems to be a natural result for $f_{b} / f_{c}$ to approach unity near the Sun.

Considering that $f_{b}$ correlates with all three of $f_{c}, f_{d}$, and $f_{\rho}$, we cannot constrain the physical mechanisms that relate to the spectral break. For instance, the observations of Vech et al. (2018) suggest that magnetic reconnection may disrupt the inertial cascade (Mallet et al. 2017) at a disruption scale that has a similar scaling to the cyclotron resonant scale if proton and electron temperatures are similar. Due to our current lack of electron temperatures, we have not made any attempt to distinguish the disruption scale.

Near the Sun, the interpretation of the spectral break should be taken carefully. One reason is the failure of the Taylor hypothesis. Our result of the modified Taylor hypothesis from Klein et al. (2015) is only available for the outwardpropagating fluctuations in the turbulence dominant with the outward-propagating components. Whether this modification is still available at the future perihelions is still unknown. Another reason is that the large amplitude fluctuations of magnetic fields and proton bulk velocities are found prevalent near the Sun (Bale et al. 2019; Kasper et al. 2019). The generation and the role of these structures in the solar wind turbulence is an open question. In this paper, these fluctuations are treated as a part of the turbulent cascade. The behavior of the spectral break in these structures need further elucidation.

As PSP descends deeper into the heliosphere, we expect to study the break scale where physical scales show better separation in the spacecraft frequency. In addition to studying the spectral break, investigation into the dynamics of particles 

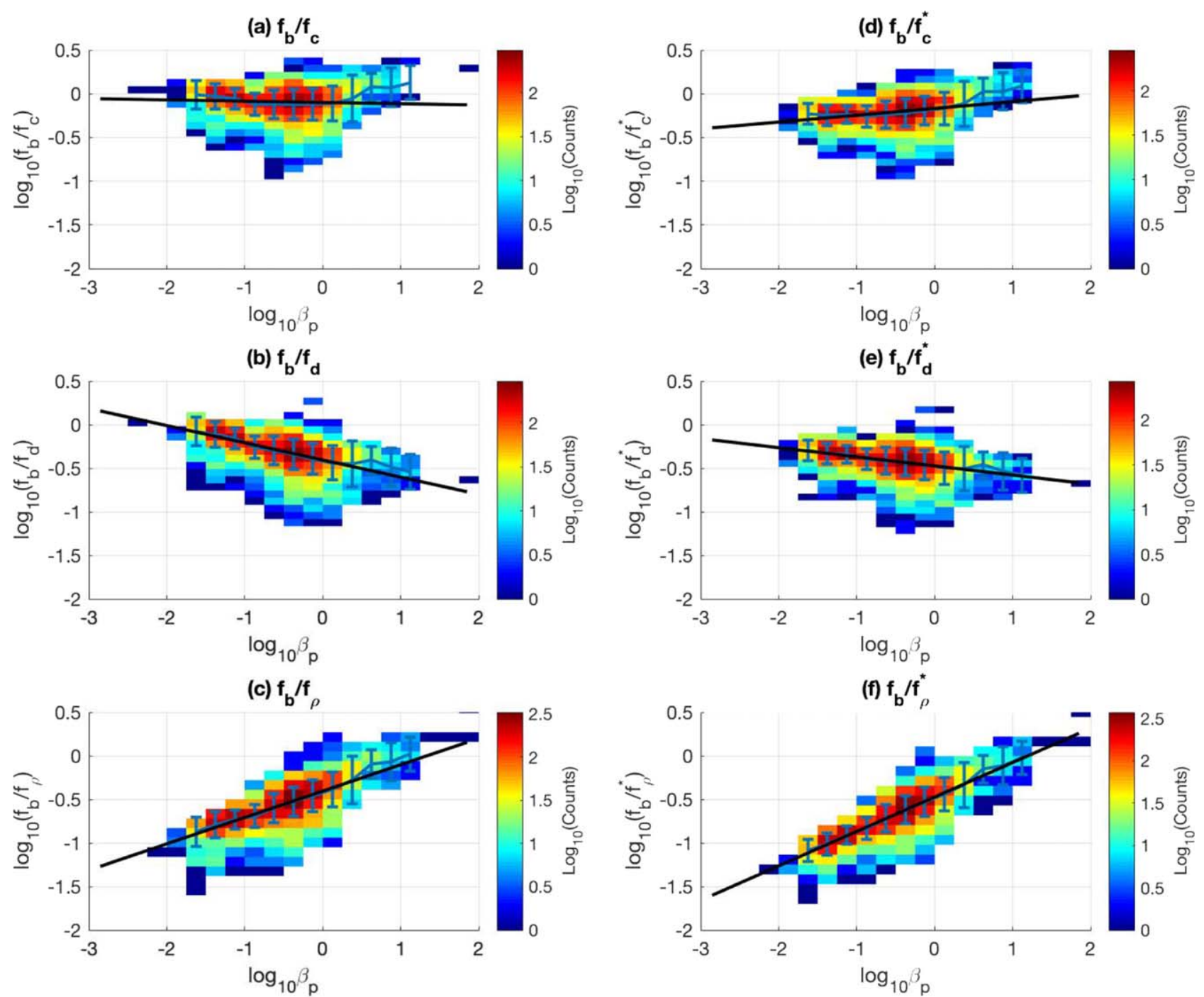

Figure 6. The 2D-histogram of the distribution of the occurrence of (a) $\log _{10}\left(f_{b} / f_{c}\right)$, (b) $\log _{10}\left(f_{b} / f_{d}\right),\left(\right.$ c) $\log _{10}\left(f_{b} / f_{\rho}\right),\left(\right.$ d) $\log _{10}\left(f_{b} / f_{c}^{*}\right),\left(\right.$ e) $\log _{10}\left(f_{b} / f_{d}^{*}\right), \operatorname{and}(\mathrm{f})$ $\log _{10}\left(f_{b} / f_{\rho}^{*}\right)$ over $\beta_{p}$, respectively. The starred frequencies are the corresponding frequencies from the modified Taylor hypothesis. The black lines are the linear fitting with least-square method. The averages and standard deviations of each $\beta_{p}$ bin are plotted as blue lines.

Table 2

Summary of the Correlation Coefficients of Various Power-law Fits

\begin{tabular}{cccc}
\hline \hline Parameter 1 & Parameter 2 & PCC & SCC \\
\hline$f_{b}$ & $r$ & -0.81 & -0.84 \\
& $v_{\mathrm{sw}}$ & 0.11 & 0.10 \\
\hline \multirow{3}{*}{$f_{b}$} & $\beta_{p}$ & -0.45 & -0.51 \\
\hline & $f_{c}$ & 0.78 & 0.76 \\
& $f_{d}$ & 0.70 & 0.64 \\
$r$ & $f_{\rho}$ & 0.69 & 0.72 \\
\hline & $f_{b} / f_{c}$ & -0.40 & -0.34 \\
& $f_{b} / f_{d}$ & -0.61 & -0.63 \\
& $f_{b} / f_{\rho}$ & 0.13 & 0.30 \\
\hline & $f_{b} / f_{c}^{*}$ & -0.07 & -0.03 \\
& $f_{b} / f_{d}^{*}$ & -0.40 & -0.39 \\
& $f_{b} / f_{\rho}^{*}$ & 0.32 & 0.47 \\
\hline$\beta_{p}$ & $f_{b} / f_{c}$ & -0.05 & -0.09 \\
& $f_{b} / f_{d}$ & -0.55 & -0.59 \\
\hline & $f_{b} / f_{\rho}$ & 0.71 & 0.71 \\
\hline$\beta_{p}$ & $f_{b} / f_{c}^{*}$ & 0.27 & 0.27 \\
& $f_{b} / f_{d}^{*}$ & -0.36 & -0.38 \\
& $f_{b} / f_{\rho}^{*}$ & 0.82 & 0.82 \\
\hline & & &
\end{tabular}

and waves at kinetic scales may constrain the process by which the spectra steepens. The observational studies find that the kinetic fluctuations could be quasi-parallel ion cyclotron waves, quasi-perpendicular KAW, or the combination of both types at 1 au (He et al. 2011, 2012a, 2012b; Salem et al. 2012; Klein et al. 2014; Zhao et al. 2017). The behavior of the fluctuation near the break scale in the inner heliosphere needs a more comprehensive analysis. As the evidences of the magnetic reconnection and accompanying turbulent enhancement are found in the solar wind (Gosling et al. 2005; Phan et al. 2006; $\mathrm{He}$ et al. 2018), the kinetic-scale fluctuation from the reconnection is another possible explanation of the spectral break. The contribution of the reconnection comparing with other mechanisms requires quantitative clarification.

We thank the referee for helpful comments and the NASA Parker Solar Probe Mission and the FIELDS and SWEAP teams for use of data. D.D. is supported by the China Scholarship Council for his stay at SSL. C.H.K.C. is supported by STFC Ernest Rutherford Fellowship ST/N003748/2. The FIELDS and the SWEAP experiment on the Parker Solar 
Probe spacecraft was designed and developed under NASA contract NNN06AA01C. D.D. and J.S.H. are also supported by NSFC under 41874200, 41574168, and 41421003. The authors acknowledge the extraordinary contributions of the Parker Solar Probe mission operations and spacecraft engineering teams at the Johns Hopkins University Applied Physics Laboratory. PSP data is available on SPDF (https://cdaweb. sci.gsfc.nasa.gov/index.html/).

\section{ORCID iDs}

Trevor A. Bowen (1D https://orcid.org/0000-0002-4625-3332 Christopher H. K. Chen (1) https://orcid.org/0000-00034529-3620

Jiansen He (10) https://orcid.org/0000-0001-8179-417X Stuart D. Bale (i) https://orcid.org/0000-0002-1989-3596

Daniel Vech (1) https://orcid.org/0000-0003-1542-1302

J. C. Kasper (iD https://orcid.org/0000-0002-7077-930X

Marc Pulupa (iD https://orcid.org/0000-0002-1573-7457

Anthony W. Case (iD https://orcid.org/0000-0002-3520-4041

Kelly E. Korreck (1) https://orcid.org/0000-0001-6095-2490

Robert J. MacDowall (1D https://orcid.org/0000-0003-

3112-4201

David M. Malaspina (1D https://orcid.org/0000-0003-

1191-1558

Phyllis Whittlesey (iD https://orcid.org/0000-0002-7287-5098

\section{References}

Alexandrova, O., Saur, J., Lacombe, C., et al. 2009, PhRvL, 103, 165003 Bale, S. D., Badman, S. T., Bonnell, J. W., et al. 2019, Natur, 576, 237

Bale, S. D., Goetz, K., Harvey, P. R., et al. 2016, SSRv, 204, 49

Bale, S. D., Kellogg, P. J., Mozer, F. S., Horbury, T. S., \& Reme, H. 2005, PhRvL, 94, 215002

Bourouaine, S., Alexandrova, O., Marsch, E., \& Maksimovic, M. 2012, ApJ, 749, 102

Bourouaine, S., \& Chandran, B. D. 2013, ApJ, 774, 96

Bourouaine, S., \& Perez, J. C. 2018, ApJL, 858, L20

Bourouaine, S., \& Perez, J. C. 2019, ApJL, 879, L16

Bowen, T. A., Mallet, A., Huang, J., et al. 2020, ApJS, doi:10.3847/15384365/ab6c65

Bowen, T. A., Mallet, A., Bonnell, J. W., \& Bale, S. D. 2018, ApJ, 865, 45

Bruno, R., \& Carbone, V. 2013, LRSP, 10, 2

Bruno, R., \& Trenchi, L. 2014a, ApJL, 787, L24

Bruno, R., Trenchi, L., \& Telloni, D. 2014b, ApJL, 793, L15

Chandran, B. D., Li, B., Rogers, B. N., Quataert, E., \& Germaschewski, K. 2010, АpJ, 720, 503

Chen, C. H. K. 2016, JPIPh, 82, 535820602

Chen, C. H. K., Bale, S. D., Bonnell, J. W., et al. 2020, ApJS, doi:10.3847/ $1538-4365 / \mathrm{ab} 60 \mathrm{a} 3$

Chen, C. H. K., Horbury, T. S., Schekochihin, A. A., et al. 2010, PhRvL, 104, 255002
Chen, C. H. K., Leung, L., Boldyrev, S., Maruca, B. A., \& Bale, S. D. 2014, GeoRL, 41, 8081

Chhiber, R., Usmanov, A. V., Matthaeus, W. H., Parashar, T. N., \& Goldstein, M. L. 2019, ApJS, 242, 12

Cho, J., \& Vishniac, E. T. 2000, ApJ, 539, 273

Dmitruk, P., Matthaeus, W. H., \& Seenu, N. 2004, ApJ, 617, 667

Duan, D., He, J., Pei, Z., et al. 2018, ApJ, 865, 89

Fox, N. J., Velli, M. C., Bale, S. D., et al. 2016, SSRv, 204, 7

Franci, L., Landi, S., Matteini, L., Verdini, A., \& Hellinger, P. 2016, ApJ, 833, 91

Galtier, S. 2006, JPIPh, 72, 721

Gosling, J. T., Skoug, R. M., McComas, D. J., \& Smith, C. W. 2005, JGRA, 110,1107

He, J., Marsch, E., Tu, C., Yao, S., \& Tian, H. 2011, ApJ, 731, 85

He, J., \& Tian, H. 2019, Sci. China Tech. Sci., 62, 1481

He, J., Tu, C., Marsch, E., Bourouaine, S., \& Pei, Z. 2013, ApJ, 773, 72

He, J., Tu, C., Marsch, E., \& Yao, S. 2012a, ApJL, 745, L8

He, J., Tu, C., Marsch, E., \& Yao, S. 2012b, ApJ, 749, 86

He, J., Zhu, X., Chen, Y., et al. 2018, ApJ, 856, 148

Horbury, T. S., Forman, M., \& Oughton, S. 2008, PhRvL, 101, 175005

Howes, G. G. 2017, PhPl, 24, 055907

Howes, G. G., Cowley, S. C., Dorland, W., et al. 2008, JGRA, 113, A05103

Kasper, J. C., Bale, S. D., Belcher, J. W., et al. 2019, Natur, 576, 228

Kasper, J. C., Abiad, R., Austin, G., et al. 2016, SSRv, 204, 131

Kiyani, K. H., Osman, K. T., \& Chapman, S. C. 2015, RSPTA, 373, 20140155

Klein, K. G., Howes, G. G., TenBarge, J. M., \& Podesta, J. J. 2014, ApJ, 785, 138

Klein, K. G., Perez, J. C., Verscharen, D., Mallet, A., \& Chandran, B. D. 2015, ApJL, 801, L18

Leamon, R. J., Matthaeus, W. H., Smith, C. W., et al. 2000, ApJ, 537, 1054

Leamon, R. J., Smith, C. W., Ness, N. F., Matthaeus, W. H., \& Wong, H. K. 1998, JGR, 103, 4775

Leamon, R. J., Smith, C. W., Ness, N. F., \& Wong, H. K. 1999, JGRA, 104, 22331

Loureiro, N. F., \& Boldyrev, S. 2017, ApJ, 850, 182

Mallet, A., Schekochihin, A. A., \& Chandran, B. D. G. 2017, JPIPh, 83 , 905830609

Markovskii, S., Vasquez, B. J., \& Smith, C. W. 2008, ApJ, 675, 1576

Marsch, E. 2006, LRSP, 3, 1

Martinović, M. M., Klein, K. G., \& Bourouaine, S. 2019, ApJ, 879, 43

Narita, Y., Glassmeier, K.-H., Motschmann, U., \& Wilczek, M. 2013, EP\&S, 65,5

Perri, S., Carbone, V., \& Veltri, P. 2010, ApJL, 725, L52

Gary, S. P., \& Smith, C. W. 2009, JGRA, 114, A12105

Phan, T. D., Gosling, J. T., Davis, M. S., et al. 2006, Natur, 439, 175

Salem, C. S., Howes, G. G., Sundkvist, D., et al. 2012, ApJL, 745, L9

Schekochihin, A. A., Cowley, S. C., Dorland, W., et al. 2009, ApJS, 182, 310

Stawicki, O., Gary, S. P., \& Li, H. 2001, JGRA, 106, 8273

Vasquez, B. J., Abramenko, V. I., Haggerty, D. K., \& Smith, C. W. 2007, JGRA, 112, A11102

Vech, D., Klein, K. G., \& Kasper, J. C. 2017, ApJL, 850, L11

Vech, D., Mallet, A., Klein, K. G., \& Kasper, J. C. 2018, ApJL, 855, L27

Verscharen, D., Klein, K. G., \& Maruca, B. A. 2019, LRSP, 16, 5

Wang, X., Tu, C. Y., He, J. S., \& Wang, L. H. 2018, JGRA, 123, 68

Woodham, L. D., Wicks, R. T., Verscharen, D., \& Owen, C. J. 2018, ApJ, 856,49

Yan, L., He, J., Zhang, L., et al. 2016, ApJL, 816, L24

Zhao, G. Q., Chu, Y. H., Lin, P. H., et al. 2017, JGRA, 122, 4879 